\title{
Slogans publicitaires et l'art de rester précisément imprécis
}

\author{
Silvia Adler ${ }^{1, *}$ \\ ${ }^{1}$ Université Bar-Ilan, Israël
}

\begin{abstract}
Résumé. Dans la lignée de mes travaux précédents sur la syntaxe et la sémantique des slogans publicitaires, d'une part, et sur la précision et l'imprécision, d'autre part, je m'interroge, dans la présente étude, sur la valeur de l'imprécis dans les slogans publicitaires en plus. Pourquoi opter pour l'imprécis et le vague dans la démarche de promotion commerciale, alors que le slogan serait en principe censé communiquer de façon compacte un message tranchant permettant à la marque de se distinguer et aux destinataires de capter les avantages du service ou du produit promus dans un laps de temps très court? Quel serait le rôle de l'imprécis dans la stratégie promotionnelle?
\end{abstract}

\begin{abstract}
Advertising slogans and the art of remaining precisely imprecise. In line with my previous work on the syntax and semantics of advertising slogans, on the one hand, and on accuracy and vagueness, on the other hand, the present paper inquires into the value of vagueness and imprecision in advertising slogans carrying the French adverb plus (eng., more). Why opting for vagueness and imprecision in the process of commercial promotion? Isn't a slogan supposed to communicate a compact and sharp message allowing the brand to distinguish itself and addressees to capture the benefits of the service or the product promoted in a very short time? What roles do vagueness and imprecision play in the promotional strategy?
\end{abstract}

\section{Introduction}

Dans la lignée de mes travaux précédents sur la syntaxe et la sémantique des slogans publicitaires (Adler, 2015, 2018a, 2018b), d'une part, et sur la précision et l'imprécision, d'autre part (Bat-Zeev Shyldkrot, Adler \& Asnes (éds), 2010, 2014, 2016), je m'interroge, dans la présente étude, sur la valeur de l'imprécis dans les slogans publicitaires (accroches ou signatures) en plus. Plus précisément, je m'intéresse à la question de savoir pourquoi opter pour l'imprécis et le vague dans la démarche de promotion commerciale, alors que le slogan serait en principe censé communiquer de façon compacte un message tranchant permettant à la marque de se distinguer et aux destinataires de capter les avantages du service ou du produit promus dans un laps de temps très court (néanmoins, de retenir le message pour une période aussi longue que possible) $)^{\mathrm{i}}$.

\footnotetext{
* Corresponding author : silvia.adler@biu.ac.il
} 
Fondée sur la base Slogansdepub ${ }^{\mathrm{ii}}$ qui répertorie plus de 4,000 slogans au jour du 28 juillet 2019, mon étude procédera en deux temps : il s'agira, tout d'abord, de cataloguer les différentes valeurs de plus dans les 244 slogans portant cet item et retrouvés dans la base $\mathrm{e}^{\mathrm{iii}}$. Après avoir écarté les occurrences de plus qui ne dénotent pas l'imprécis, on s'interrogera, dans un deuxième temps, sur la relation de plus à l'imprécis et sur sa valeur stratégique dans l'acte de promotion.

\section{Valeurs de plus dans les slogans répertoriés}

Les résultats obtenus révèlent que toutes les occurrences de plus ne conduisent pas à une imprécision (voir section 2.2), mais aussi que lorsque plus engendre une imprécision, celleci n'est pas homogène. En voici les principales valeurs, illustrées par une sélection de slogans dans la section 2.1 qui suit.

\subsection{Plus et l'imprécis}

\subsubsection{Intensification d'un verbe matérialisé ou non}

(1a) 5àsec — pressings : Lavez moins, vivez plus.

(1b) Albal - emballages alimentaires : Demandez-nous toujours plus.

(1c) Axe - produits d'hygiène : Plus t'en mets, plus t'en as.

(1d) Bic — produits de papeterie, etc. : Plus pour votre argent... Toujours !

(1e) Bouygues Immobilier : Bien plus pour vous.

(1f) Carte d'Or : Offrez plus, offrez Carte d'Or.

(1g) Dacia automobiles : Exigez plus, payez moins.

(1h) Dell — produits électroniques : Le pouvoir d'en faire plus.

(1i) Eram - magasins de chaussures : Il faudrait être fou pour dépenser plus.

(1j) Fer-Plus — pressing : Fer Plus, c'est faire Mieux ! iv $^{\text {in }}$

Ces exemples illustrent une situation où le verbe, matérialisé (par ex. dans (1a) et (1b)) ou non (par ex. dans (1d) et (1e)) $)^{\mathrm{v}}$ est intensifié par plus (et, éventuellement, un complément non matérialisévi ${ }^{\mathrm{vi}}$. L'adverbe, qui vient modifier la prédication, met en valeur l'avantage du produit ou du service promus. Ainsi, la possibilité de vivre, demander ou exiger plus constitue un avantage direct pour le client potentiel. Par ailleurs, une compagnie qui est capable d'offrir plus est retenue comme une compagnie solide et bien établie, ce qui éveille la confiance de la clientèle potentielle. Dans (1i), dépenser plus représente une situation désavantageuse pour le client potentiel, mais étant donné que cette situation est liée à une éventualité - et donc à une contrefactualité - le message obtenu est, au contraire, que le client est une personne pas comme les autres et que son comportement économique est tout à fait rationnel.

L'imprécis dans ces slogans est lié au fait que la grande quantité (beaucoup) ou un degré plus haut, à savoir un dépassement (davantage) par rapport à un seuil sont des valeurs relatives en ce sens d'une impossibilité de déterminer ou de fixer leur application référentielle ${ }^{\mathrm{vii}}$.

\subsubsection{Modification d'un adjectif}

(2a) Airness - articles de sport : Toujours plus haut, toujours plus fort !

(2b) Arthur Martin Electrolux - électroménagers : Pour une vie plus facile.

(2c) BaByliss - petits électroménagers : Plus belle plus vite. 
(2d) Bricoman - magasins de bricolage : Plus pro, moins cher.

(2e) Cetelem - crédits à la consommation : Plus responsables, ensemble.

(2f) Champion - supermarchés - devenu Carrefour : Plus facile la vie.

(2g) Euro Millions - loterie : Et si votre vie devenait infiniment plus riche ?

(2h) Financière Sun Life : La vie est plus radieuse sous le soleil.

(2i) France 2 - chaîne de télévision : L'air est plus vif sur France 2.

(2j) Saturn : Plus radin, plus malin.

(2k) Simply Market : Plus proche de vous.

Dans cette catégorie, sont regroupés des slogans où l'adverbe plus modifie un adjectif. Je fais abstraction de la séquence toute entière, celle-ci peut être de nature variée: comparaisons tronquées ${ }^{\text {viii }}$, séquences averbales, parataxes asyndétiques, thématisation du groupe adjectival, etc. En matière d'imprécis, on ajoutera - outre les faits que l'adjectif est scalaire et que plus modifie l'intensité de celui-ci sans pourtant limiter sa portée - le fait que plus présuppose l'existence d'un autre élément qui est effectivement $X$ (la valeur dénotée par l'adjectif) ou moins $X$ (une valeur amoindrie). Cet élément peut référer aux concurrents, ou à un état relatif au bien-être du consommateur potentiel, avant la consommation du produit ou du service promus. Quoi qu'il en soit, le message publicitaire ne procure pas de précisions quant au comparé ; il met plutôt en avant le fait de dépasser un standard ou un statu quo, dépassement qui n'est pas déterminé non plus, puisque l'échelle d'intensité n'est pas bornée.

\subsubsection{Plus que + Nom / Infinitif}

(3a) Banque Postale (la) : Bien plus qu'une banque.

(3b) EDF - Électricité de France : Nous vous devons plus que la lumière.

(3c) Europcar : Vous louez plus qu'une voiture.

(3d) Seafrance : Bien plus que traverser la Manche.

(3e) Autoroute Info : Plus qu'une radio, un compagnon de route.

(3f) ONG Conseil : Plus qu'un job, une expérience de vie !

Les slogans (3e) et (3f) diffèrent des slogans (3a)-(3d), en ce qu'ils contiennent une entité dédoublée et ellipsée (autoroute info / ONG Conseil), deux paramètres (être radio et être compagnon de route / être un job et être une expérience de vie) figurant respectivement dans chacun des deux slogans - dans une proposition conjonctive averbale et dans une proposition principale averbale. Il n'y a pas de standard de comparaison mais on en déduit dans $(3 \mathrm{e})$ que les autres radios ne possèdent que le paramètre d'être radio, sans rien de plus, donc ne se distinguent pas les unes des autres et ne font par ailleurs aucun effort pour se distinguer (elles ne font que ce qu'elles sont censées faire, sans rien de plus). Idem pour (3f) qui laisse comprendre que les autres services de conseil ne procurent que le paramètre qu'ils sont censés procurer de toute façon et rien à part ce paramètre.

De surcroît, à côté de la lecture standard, ces slogans donnent accès à une comparaison épistémique (Fuchs, 2010 : 2091), qui « opère au niveau 'métalinguistique' et non pas au niveau prédicatif $»$ ix . Voici une illustration de ces deux lectures pour le slogan (3e) :

(3ei). (comparaison standard) sa capacité d'être compagnon de route est plus grande que sa capacité en tant que radio ;

(3eii). (comparaison épistémique) il est plus exact / juste / pertinent de dire que Autoroute Info est un compagnon de route que de dire que c'est une simple radio.

Plusieurs indices caractéristiques étayent la possibilité d'une lecture épistémique, dont la prédication de deux qualités à la même entité ; la nature averbale de la comparaison; la 
nature non graduable des qualités prédiquées; la possibilité de déplacer le marqueur comparatif suivi de la subordonnée : «Autoroute Info, plus qu'une radio est un compagnon de route » / «Autoroute Info est un compagnon de route, plus qu'une radio » / « Plus qu'une radio, Autoroute Info est un compagnon de route » / «Autoroute Info est un compagnon de route, plutôt qu'une radio ». Selon Fuchs (2010 : 2093) " une telle segmentation de la phrase pousse en effet à assigner une portée extraprédicative au quantificateur ; et donc à y voir, non pas une mesure du degré des qualités (lecture standard), mais une mesure du degré d'adéquation au réel des assertions (d'où une lecture épistémique) ».

L'exemple (3e) opère une disjonction inclusive entre les deux assertions (Fuchs, 2010 : 2097), voire un cas de figure où «l'un des deux contenus propositionnels présente un degré d'adéquation au réel supérieur à l'autre, mais les deux sont donnés comme susceptibles d'être vrais ensemble », étant donné que les deux perspectives sont non mutuellement exclusives. Là réside donc l'avantage de cette chaîne radio qui, tout en étant radio et en remplissant toutes les fonctions qu'une chaîne radio est censée remplir, elle fait quelque chose de plus (adéquat, pertinent, favorable) qui peut être vrai en même temps.

En comparaison avec (3e) et (3f), les slogans (3a) - (3d) laissent encore plus de liberté de reconstruction mentale, puisqu'ils ne mentionnent que le dépassement d'un standard ou d'un seuil inférieur, sans rien de plus. Dans le cas de (3a), par exemple, que signifie être plus qu'une banque ? En quoi consiste le dépassement ? Cet 'avantage' n'est précisé, ni en termes quantitatifs ni en termes qualitatifs.

\subsubsection{Modification d'un adverbe}

(4a) Belin — snacks : Et si on prenait l'apéritif plus souvent?

(4b) Dunlopillo - literie : Vous dormirez plus $\operatorname{tard}^{\mathrm{x}}$.

(4c) Ford : Allons plus loin / Go Further.

(4d) Ordinateur Individuel (l') : Allez plus loin dans le numérique.

(4e) Calgon : Le lave-linge dure plus longtemps avec Calgon!

Plus intensifie le pouvoir de l'adverbe dans le contexte d'une prédication donnée et consolide davantage cette prédication (prendre l'apéritif, durer, etc.), d'où l'avantage du produit ou du service promus, qui consiste dans le dépassement d'un seuil ou un standard relatifs à un produit, un service ou une activité quotidienne. Tout en opérant une intensification, l'échelle reste néanmoins ouverte en ce sens que la limite supérieure n'est pas spécifiée ou garantie. Il va sans dire qu'aucune précision n'est donnée non plus quant au seuil inférieur. Le fait de prendre l'apéritif, durer, etc. est donné comme présupposé.

\subsubsection{Varia}

(5a) Canal+ : Et tellement + encore.

(5b) Fanta : Plus c'est Fanta, moins c'est sérieux.

(5c) France 2 : Plus 2 passion.

(5d) Nintendo : Plus c'est intelligent, plus c'est amusant.

(5e) Scores (rôtisseries) : Toujours plus chez Scores !

(5f) Chevrolet - automobiles : Un vrai plus !

Du point de vue de la configuration syntaxique, on trouve ici des structures comparatives en corrélation (plus...plus.../ plus... moins...) ou des séquences averbales. Plus figure en emploi adverbial (toujours plus), substantif (Un vrai plus) ou déterminatif (plus de (2) Nom).

Le dénominateur commun à tous ces emplois est le fait d'une modification vers le haut d'une quantité ou d'une qualité. Ce supplément consiste dans un avantage pour les clients 
potentiels. Le message reste pourtant imprécis du fait d'une impossibilité d'attribuer une valeur déterminée à plus et donc d'arrêter la valeur référentielle de l'intensification.

\subsection{Autres}

\subsubsection{Superlatif : le plus (+Adjectif)}

(6a) Action Man - figurines : Le plus grand de tous les héros.

(6b) Air France : Faire du ciel le plus bel endroit sur Terre.

(6c) Bluewater Seafoods : Les idées les plus rafraîchissantes pour les fruits de mer.

(6d) Canal+ : Le plus de la télévision.

(6e) Chante France : Les plus belles chansons françaises.

(6f) Club Internet - devenu SFR : Le club le plus ouvert de la planète.

(6g) Scorpio : Le plus brûlant des parfums d'homme.

Le superlatif de supériorité présuppose une comparaison entre au moins deux membres, mais il désigne en même temps que le comparé a atteint le plus haut degré possible (le degré extrême) parmi les autres membres pris en compte dans la comparaison (Riegel, Pellat et Rioul, 1996 : 365-366). Autrement dit, le superlatif cherche à fermer l'échelle de degrés pour transmettre un message de perfection ou une précision métaphorique. De ce fait, j'écarte ces exemples de mon analyse.

\subsubsection{Plus : adverbe de négation}

(7a) Bis Télévisions : Le satellite n'est plus astronomique.

(7b) Ekolux - pierre et carrelage : Le luxe n'est plus un luxe !

(7c) Houra.fr - supermarché en ligne : Et si vous passiez votre temps à ne plus faire les courses.

Que la négation soit descriptive ou qu'elle constitue le rejet d'une assertion ou d'une idée en cours (donc, argumentative), le slogan portant l'adverbe négatif plus asserte la non correspondance du contenu propositionnel à une réalité temporelle quelconque. Pour Muller, ce « quantifieur aspectuel » $(1991: 290-291)$ constitue la version négative d'encore. Selon Riegel, Pellat et Rioul (1996: 418), plus «indique la rupture d'une continuité temporelle. Il découpe la succession temporelle en distinguant un avant et un après [...]. Il donne à l'avant un statut de présupposé $»$.

Là, réside donc l'avantage du produit ou du service promus : le fait d'annuler un état passé, conçu comme désavantageux pour le destinataire. Ces exemples seront écartés de l'analyse qui suit. Non que l'imprécis y soit totalement absent, puisqu'il concerne bel et bien le plan des implicatures (passer son temps à ne plus faire les courses n'asserte pas comment chacun pourrait désormais remplir son temps, une fois libéré de ladite tâche), plutôt parce que plus ne constitue pas en tant que tel, comme dans les exemples de la section 2.1 qui précède, l'essentiel de l'avantage quantitatif et /ou qualitatif.

\section{L'imprécision en plus}

À présent, il s'agira de vérifier dans quelle mesure plus participe à la construction de l'imprécis (§ 3.1) pour dégager - ultérieurement (\$3.2) - la valeur stratégique de plus dans le message commercial. 


\subsection{Quelques précisions sur l'imprécision, en général, et sur celle en plus, en particulier}

Le TLFi définit imprécis comme suit: "dont la nature, plus ou moins indifférenciée, est vague, difficile à identifier, à définir ». Cette définition générale sera ajustée pour être rendue conforme à différents contextes. Ainsi, dans le domaine de la perception visuelle, la définition proposée est «dont la forme ou les contours manquent de netteté », alors que dans le domaine de la perception auditive, le TLFi propose « dont la nature et /ou l'origine sont difficilement reconnaissables ». "Dont la cause et /ou l'objet restent indéterminés, difficiles à cerner » est la définition donnée pour couvrir le domaine des états d'âme et des sentiments, et «dont la formulation ou la présentation sont incomplètes, lacunaires, insuffisamment explicites » est proposé pour référer au domaine conceptuel. Pour le domaine gestuel, le $T L F i$ propose finalement «qui manque de précision, qui s'effectue de manière peu sûre ».

C'est précisément pour rendre compte de phénomènes qui appartiennent à la logique floue que Lakoff (1973a \& b) avait développé la grammaire floue. Comme beaucoup d'autres chercheurs l'ont montré depuis (Kleiber et Riegel, 1978 ; Jayez, 1987 ; Kleiber, 1987 ; Whittaker, 2002 ; Lupu, 2003 ; Mihatsch, 2007, 2010 ; Adler et Asnes 2010, 2014 ; Krifka, 2012), il s'agit de phénomènes qui ne peuvent pas être décrits par une logique binaire ne tenant compte que du vrai et du faux : entre le vrai et le faux, ou entre 0 et 1 il existe plusieurs valeurs correspondant à plusieurs degrés de vérité. Un dérivé de cette logique est qu'une entité peut appartenir 'plus ou moins' à un ensemble contenant (une classe, une catégorie), à la différence d'une situation dichotomique d'appartenance ou d'exclusion totales. Entre les deux pôles extrêmes il y a une échelle de degrés d'application ou d'appartenance et il est aussi possible de considérer les faits selon une hiérarchie ( + / -) d'appartenance.

Pour Mihatsch (2010: 125), « de nombreux domaines sémantiques [...], s'organisent sous forme d'échelles. Les concepts ou les différentes valeurs d'un concept se positionnent alors de façon linéaire et progressive et souvent orientés vers un extrême de l'échelle ". Entre autres, elle cite le domaine de la quantification ou celui de la télicité. Krifka (2012) considère les adjectifs scalaires (graduables) comme un cas prototypique de l'expression du vague étant donné que leur interprétation dépend d'une comparaison (souvent implicite). Pour cet auteur, le vague (l'imprécis) est un phénomène pragmatique qui «arises as a phenomenon of applying the literal meaning of expressions in communication ».

En ce qui concerne plus de façon spécifique, les slogans de la section précédente montrent tous que le flou ou l'imprécis reposent sur un jugement de valeur, et donc sur l'impossibilité d'évoquer une valeur (standard) précise, stable, durable ou préétablie. Plus a beau désigner une orientation (ascendante) sur une échelle, toujours est-il qu'il n'indique pas l'endroit précis dans l'échelle. Qui plus est, l'échelle en question peut ne pas être fermée à son extrême supérieur, sans mentionner le fait que nous ne connaissons pas toujours le seuil inférieur. Dans la comparaison en plus, le comparant (souvent implicite) sert de point de référence et le comparé en plus se voit dans une position 'supérieure' par rapport à ce point de référence (Fauconnier, 1984 : 167-169), mais que signifie alors 'supérieur' ? En effet, les slogans en plus construisent une comparaison (souvent implicite) de supériorité, donc un sous-type de comparaison d'inégalité (Rivara, 1990 et 1995 ; Fuchs, 2014), laquelle non seulement met en valeur l'écart et la différence entre entités comparées (les comparandes), mais aussi instaure une gradation, selon une échelle ascendante, orientée positivement.

\subsection{Avantages de l'imprécision en plus}


La question de la valeur de plus dans le slogan s'impose puisque l'on pourrait s'attendre légitimement à ce qu'un message commercial et promotionnel soit tranchant et décisif. Quel(s) serai(en)t donc le(s) rôle(s) de plus ? Reprenons quelques exemples des sections précédentes $(\$ 2.1 .1$ - 2.1 .5$)$ pour découvrir la valeur de plus en action.

Dans les slogans de la section 2.1.1, l'imprécis communique une quantité ou une qualité (par exemple, (1a) et (1e)) qui surpasse un standard. Dans certains cas, ce standard représente une pratique courante liée au destinataire $((1 b))$, alors que dans d'autres, ce standard présuppose ce que la concurrence fait d'habitude. En tout état de cause, le message ne communique pas de valeurs quantitatives ou qualitatives fixes ou durables, mais le fait même d'augmenter ou d'intensifier la prédication constitue un dépassement du standard et donc une amélioration générale par rapport à un état de choses actuel. Si avant ou avec d'autres services ou produits le destinataire obtenait $[\mathrm{X}]$, à présent il peut obtenir $[\mathrm{X}+]$. En accord avec l'étude sociologique de Lee sur le slogan et la communication publicitaire en général (2014), on pourrait dire que l'imprécis serait un de ces procédés sémantiques qui contribuent au caractère dynamique et «sui generis » du slogan (p. 384), du fait d'une modalisation situationnelle ou contextuelle.

En ce qui concerne les slogans de la rubrique 2.1.2, le segment en plus opère, là aussi, une comparaison par rapport à un état passé ou par rapport aux concurrents. Ainsi, (2i) L'air est plus vif sur France 2, est une structure de comparaison tronquée. La troncation augmente davantage la part de l'imprécis en ce qu'elle ouvre la voie à plusieurs formules de reconstruction ou de décodage, ce qui permet de personnaliser le message et de le rapporter au monde des croyances ou à l'expérience de destinataires variés. Plus précisément, l'effacement du standard permet à ce slogan de s'adresser tant aux spectateurs des chaînes de télévision concurrentes qu'aux consommateurs des médias divers (Internet, radio, etc.) :

(2i'). L'air est plus vif sur France 2 [= qu'il ne l'est sur d'autres chaînes de télévision / sur d'autres médias / ailleurs]

En d'autres mots, réduit, le slogan atteint un but multiple, en construisant un appel soit à changer les habitudes d'audience télévisuelle, soit à changer les habitudes d'audience ou de consommation de médias. Qui plus est, le fait de se concentrer sur l'avantage (plus + Adjectif) plutôt que sur l'étalon de comparaison (le comparant, le standard, qui pourrait consister dans des chaînes concurrentes), permet d'éviter une situation de diffamation. Dans les termes de Lee (2014: 375), le slogan présente tout l'avantage de "multum in parvo » (beaucoup dans un petit espace).

Les adjectifs dans les slogans de la rubrique 2.1.2 sont graduables le long d'une échelle orientée. Plus met en œuvre une comparaison d'inégalité qui "opère sur les quantités relatives d'un 'paramètre' commun » (Fuchs, 2014 : 19). Ainsi, en (2i), par exemple, c'est le paramètre vif qui est comparé du point de vue de son degré ou de son intensité. Selon Rivara (1995 : 38), la comparaison d'inégalité, paraphrasable par le verbe dépasser, se construit à partir d'un comparant qui a le «statut énonciatif d'un repère, et qui est parfois marqué comme étant une origine » et d'un comparé qui est forcément «non identique ». Toujours est-il que dans ces exemples, l'identité du comparant n'est pas précisée.

Il s'avère que la syntaxe compacte du slogan non seulement contribue à une mémorisation aisée, mais aussi - et en parfait accord avec des chercheurs tels que Adam \& Bonhomme (1997), Navarro Domínguez (2005) et Pahud (2013) - agit de telle sorte que la signification globale excède souvent la matière explicite, afin d'intensifier le pouvoir d'action du discours publicitaire sur le récepteur.

Les slogans des rubriques 2.1.3 - 2.1.5, chacun à sa manière, laissent aussi un élément non précisé. Énoncer (3a) Bien plus qu'une banque c'est laisser une partie non résolue : en quoi consiste précisément tout ce que la Banque Postale est / fait / propose en plus ? Certes, un message pareil met en avant le dépassement par rapport aux autres banques mais aussi 
par rapport aux attentes ou à l'expérience du client potentiel. En ce sens, le message fait appel au client potentiel pour que celui-ci se débarrasse de son scepticisme habituel et veuille accorder une chance à un service qui pourrait améliorer sa vie ${ }^{\mathrm{xi}}$. Mais, à nouveau, ce type de slogan reste vague en ce qui concerne le 'supplément', en termes qualitatifs et quantitatifs. Il en va de même pour le slogan (5a) de Canal+, Et tellement + encore. Dans (4e) Le lave-linge dure plus longtemps avec Calgon! le destinataire pourrait restituer mentalement «qu'il ne dure avec d'autres produits d'entretien essayés par une personne particulière », « qu'avec n'importe quel autre produit d'entretien », « dans l'absolu ».

Il s'avère donc que, dans le discours publicitaire, l'imprécision est une stratégie avantageuse de plusieurs points de vue : elle permet d'élargir la réception du slogan en ce qu'elle est 'communicante' pour divers goûts et modes de raisonnement; elle permet de contourner un risque de diffamation et donc d'éviter des problèmes légaux ; elle permet, finalement, aux procureurs des services et des produits promus de ne pas garantir au client potentiel quelque chose qui pourrait ne pas se réaliser de facto.

Rivara (1995) décrit la comparaison d'inégalité ou «non identique » en termes de "pseudonégative ». L'avantage de cette comparaison dans le secteur publicitaire est en effet la possibilité d'établir une différence, sans pourtant annuler opérativement le positionnement ou la valeur de l'adversaire, mais aussi - dans le cas de plus - sans garantir le degré exact de ce qui est promu, pour ne pas donner au slogan un caractère contractuel.

\section{Conclusion}

L'imprécis dans le slogan s'avère une manœuvre très puissante puisqu'il donne libre cours au destinataire de 'préciser' le message mentalement et donc de le personnaliser suivant son propre mode de raisonnement, ses goûts, etc. Le destinataire devient ainsi un partenaire actif et légitime du message communiqué, vu le processus cognitif de reconstruction qu'il investit. Dans le secteur publicitaire, où il s'agit souvent de s'adresser à un public aussi large que possible, les slogans qui permettent des interprétations multiples présentent donc un atout.

De ce point de vue-là, les slogans étudiés dans la présente étude se joignent aux slogans étudiés dans mes travaux précédents. À l'égal des schémas paratactiques asyndétiques (Adler, 2015, 2018b), des ceux à constructions syndétiques avec et (Adler, 2018b) et des ceux à comparaison d'inégalité fragmentaire (2018a), les slogans en plus considérés ici ouvrent la voie à une gamme d'interprétations, du fait de leur imprécision.

Mis à part le rôle de l'imprécis en tant que moteur de persuasion et de séduction - du fait que la plurivalence interprétative est corrélée à diverses possibilités de reconstruction du standard qui n'est pas dans la portée de plus - on a signalé l'importance de l'imprécis pour éviter un acte de concurrence déloyale, mais aussi pour ne pas rendre au slogan un caractère contractuel, qui pourrait entraîner, dans ce cas aussi, d'éventuels problèmes légaux.

\section{Références bibliographiques}

Adam, J.-M. \& Bonhomme, M. (1997). L'argumentation publicitaire. Paris : Nathan.

Adam, J.-M. \& Bonhomme, M. (2003). L'argumentation publicitaire. Rhétorique de l'éloge et de la persuasion. Paris : Nathan.

Adler, S. (2015). Liaisons «non marquées » de prédications dans l'accroche publicitaire. Langages, 200, 121-136.

Adler, S. (2018a). Slogans publicitaires à comparatifs de supériorité. La linguistique, 54, 39-58. 
Adler, S. (2018b). Relations de simultanéité et de consécutivité dans l'accroche publicitaire. In Aleksandrova A. et al. (eds) Consécutivité et simultanéité en linguistique, langues et parole. Vol. 2 Syntaxe et sémantique. Coll. Dixit Grammatica, L'Harmattan. pp. 15-31.

Adler, S. \& Asnes, M. (2010). Autour de la précision. L'information Grammaticale, 125, 36-43.

Adler, S. \& Asnes, M. (2014). Quantification imprécise et quantification floue : essai de précision. In Bat-Zeev Shyldkrot, H., Adler, S. \& Asnes, M. (dir.) Précis et imprécis : étude sur l'approximation et la précision. Paris : Editions Honoré Champion. pp. 25-42.

Bat-Zeev Shyldkrot, H., Adler, S. \& Asnes, M. (2010) (éds). Approximation et Précision. L'information Grammaticale, 125. Paris-Peeters.

Bat-Zeev Shyldkrot, H., Adler, S. \& Asnes, M. (2014) (éds) Précis et imprécis : étude sur l'approximation et la précision. Paris : Editions Honoré Champion.

Bat-Zeev Shyldkrot, H., Adler, S. \& Asnes, M. (2016) (éds) Nouveaux regards sur l'approximation et la précision. Paris : Editions Honoré Champion.

Cossette, C. (2001). Publicité, déchet culturel. Québec: Les Presses de l'Université de Laval.

Fauconnier, G. (1984). Espaces mentaux. Aspects de la construction du sens dans les langues naturelles. Paris : Les Editions de Minuit.

Fuchs, C. (2010). La comparaison épistémique en français moderne. In Franck Neveu et al. (éds.), Congrès Mondial de Linguistique Française. Paris : Institut de Linguistique Française. pp. 20912102.

Fuchs, C. (2014). La comparaison et son expression en français. Paris : Ophrys.

Heude, R.-P. (1993). Dictionnaire analogique de la publicité et des médias. Paris : Eyrolles.

Jayez, J. (1987). Sémantique et approximation: Le cas de presque et à peine. Linguisticae Investigationes, XI, 1, 157-196.

Kleiber, G. (1987). Quelques réflexions sur le vague dans les langues naturelles. In Mellet, S. (dir.) Etudes de linguistique générale et de linguistique latine offertes en hommage à Guy Serbat. Paris : Société pour l'Information Grammaticale. pp. 157-172.

Kleiber, G. \& Riegel, M. (1978). Les grammaires floues. In Martin, R. (dir.) La notion de recevabilité en linguistique. Paris : Klincksieck. pp. 67-123.

Krifka, M. (2012). Approximate and Precise Use of Language. Talk given in the conference Vague Language - Vague Law?, Albert-Ludwigs-Universität Freiburg, January 20-21. http://amor.cms.hu-berlin.de/ h2816i3x/Talks/VagheitFreiburg.pdf

Lakoff, G. (1973a). Hedges : A study in Meaning Criteria and the Logic of Fuzzy Concepts. Journal of Philosophical Logic, 2/ 4, 458-508.

Lakoff, G. (1973b). Fuzzy grammar and the performance/ competence Terminology game. UC Berkeley. Retrieved from https://escholarship.org/uc/item/46h7k0fk

Lee, Ch.-H. (2014). Le slogan publicitaire, dynamique linguistique et vitalité sociale : la construction d'une esthétique sociale à travers la communication publicitaire. Sociologie. Université Paul Valéry - Montpellier III, 2014. Français. NNT : 2014MON30002. HaL id : tel-01089816.

Lupu, M. (2003). Concepts vagues et catégorisation. Cahiers de linguistique française, 25, 291-304.

Mihatsch, W. (2007). The Construction of Vagueness : "Sort of" Expressions in Romance Languages. In Radden, G., Köpcke, K.-M., Berg, Th. \& Siemund, P. (dir.) Aspects of Meaning Construction. Amsterdam/ Philadelphia : John Benjamins. pp. 225-245.

Mihatsch, W. (2010). Les approximateurs quantitatifs entre scalarité et non scalarité. Langue Française, 165/1, 125-153.

Muller, Cl. (1991). La négation en français. Syntaxe, sémantique et éléments de comparaison avec les autres langues romanes. Genève : Droz.

Navarro Domínguez, F. (2005). La rhétorique du slogan : cliché, idéologie et communication. Bulletin Hispanique, 107, $n^{\circ} 1,265-282$.

Pahud, S. (2013). Slogans et proverbes : deux paroles « mythiques ». In Anscombre, J.-Cl. et al. (éds) La parole exemplaire. Paris : Armand Colin. pp. 212-226.

Riegel, M., Pellat, J.-Ch. \& Rioul, R. (1996). Grammaire méthodique du français (2 ${ }^{\mathrm{ème}}$ édition). Paris : PUF.

Rivara, R. (1990). Le système de la comparaison. Paris : Éditions de Minuit.

Rivara, R. (1995). Pourquoi il n'y a que deux relations de comparaison. Faits de langues, 5, 19-39.

Whittaker, S. (2002). La notion de gradation. Application aux adjectifs. Bern : Peter Lang.

Lexiques 
TLFi : Trésor de la langue Française informatisé, http://www.atilf.fr/tlfi, ATILF - CNRS \& Université de Lorraine. Consulté le 22 mars 2019.

\footnotetext{
${ }^{\mathrm{i}}$ Voir Adam \& Bonhomme (2003), Navarro Domínguez (2005), Pahud (2013), Lee (2014 : 91-95). Voir par ailleurs les définitions de slogan dans les dictionnaires spécialisés, comme, par exemple, celle procurée par le Dictionnaire analogique de la publicité et des médias (1993: 342): « courte phrase publicitaire vantant les mérites d'un produit ».

${ }_{\text {ii }}^{\text {http://slogansdepub.skyrock.com/ }}$

iii Une recherche des slogans contenant moins a aussi été effectuée. La requête n'a donné accès qu'à une trentaine de slogans, dont la plupart valorise l'avantage de « payer moins » et associe souvent cet adverbe à l'adjectif cher: Vivons mieux. Vivons moins cher (Auchan), Le pays où la vie est moins chère / Bien chez soi, bien moins cher (Conforama), Exigez plus, payez moins (Dacia), Dépenser moins sans aller loin (G20). Autrement dit, moins restreint tout ce qui est considéré comme désavantageux pour le destinataire, d'où l'attrait du produit ou du service promus.

iv Jeu de mots: fer-faire.

${ }^{v}$ Dans (1d) on pourrait restituer les verbes donner ou offrir, par exemple (on vous donne/ offre plus pour votre argent). Dans (1e), c'est le verbe faire qui pourrait être restitué (on fait bien plus pour vous).

${ }^{\text {vi }}$ Par exemple en (1a) plus longuement, plus intensément, etc. Dans (1b) il s'agirait de demander plus de choses.

vii Voir la section 3 pour un traitement plus élaboré de la notion d'imprécision relative à toutes les sections de 2.1 .

viii Voir Adler (2018a) pour la syntaxe des slogans comparatifs en plus.

IX Pour Fuchs (2010 : 2091), « alors que la comparaison standard instaure une égalité ou une inégalité de degré à propos de la qualité prédiquée des entités, la comparaison épistémique instaure une égalité ou une inégalité de degré à propos de l'assertion des deux contenus propositionnels (plus précisément, à propos de leur adéquation au réel, de leur exactitude, de leur pertinence) ».

x Signalons l'ambiguïté possible de cette annonce publicitaire, laquelle peut suggérer « se réveiller plus tard » (donc prolonger le déroulement interne du verbe atélique dormir, avantage quantitatif et qualitatif), ou bien «aller se coucher plus tard» (donc, prolonger l'état d'éveil avant de commencer l'activité de dormir. Les raisons pour aller se coucher plus tard seraient, elles aussi, avantageuses pour la clientèle : une belle literie pourrait influer sur nos sens et nos envies).

${ }^{x i}$ Selon Cossette (2001 : 72), « le but de la publicité n'est pas de vendre. Le rôle de la publicité, c’est d'essayer de changer l'attitude du consommateur face à un produit ou un service, de faire percevoir ceux-ci plus positivement, voire de créer une aura autour du produit, d'y ajouter une valeur symbolique ».
} 